\title{
Outcome of phacoemulsification combined with excisional goniotomy using the Kahook Dual Blade in severe glaucoma patients at 6 months
}

This article was published in the following Dove Medical Press journal: Clinical Ophthalmology

\author{
Matthew T Hirabayashi \\ Joshua T King \\ Dayeong Lee \\ Jella A An
}

Mason Eye Institute, University of Missouri School of Medicine, Columbia, MO 6520I, USA

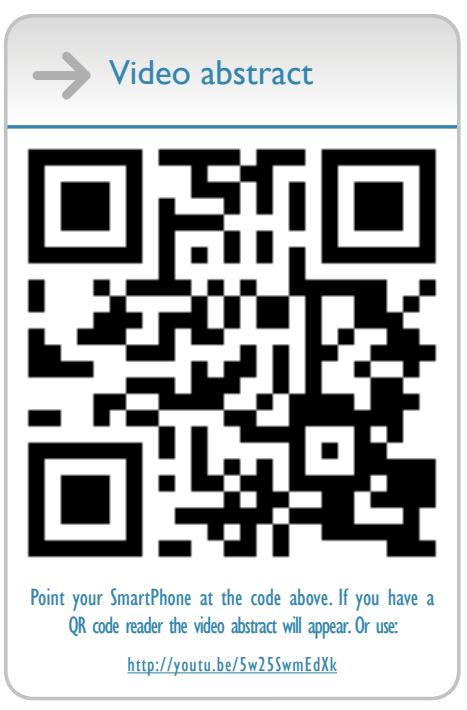

Correspondence: Jella A An Mason Eye Institute, University of Missouri School of Medicine, 3215 Wingate Court, Columbia, MO 6520I, USA

Tel +I 573884 7I56

Email anja@health.missouri.edu
Purpose: The aim of this study was to describe 6-month rates of achieving target IOP without requiring additional glaucoma surgery after excisional goniotomy using the Kahook Dual Blade combined with phacoemulsification (phaco-KDB) in patients with severe-stage glaucoma.

Methods: Retrospective review of 42 eyes from 36 patients with severe glaucoma who received phaco-KDB. Primary and secondary open-angle and combined-mechanism glaucoma were included. Preoperative, intraoperative, and postoperative data were collected through the 6-month follow-up period. The primary outcome was proportion of patients achieving IOP $\leq 15 \mathrm{mmHg}$ without additional glaucoma procedures. Secondary outcomes were mean change in IOP, reduction of glaucoma medications, additional glaucoma procedures needed, and adverse events.

Results: Preoperative baseline mean IOP was $17.1 \pm 4.8 \mathrm{mmHg}$ (mean $\pm \mathrm{SD}$ ) and number of medications was 2.4 \pm 1.3 . At 6 months, $64.3 \%$ (27/42) of eyes had achieved IOP $\leq 15 \mathrm{mmHg}$ without additional glaucoma procedures, 45.2\% (19/42) reached this target IOP on fewer medications, and $31.0 \%(13 / 42)$ on no medications. Mean IOP reduction was $2.1 \pm 4.67 \mathrm{mmHg}(P=0.022)$ and mean medication reduction was $1.2 \pm 1.4(P \leq 0.001)$. Visually significant complications were experienced by $7.1 \%$ (3/42) of eyes, and 7.1\% (3/42) required additional IOP-lowering procedures within 6 months of surgery.

Conclusion: Patients with severe-stage open-angle glaucoma achieved significant IOP and medication reductions following phaco-KDB. Notably, about two-thirds of eyes achieved an IOP of $\leq 15 \mathrm{mmHg}$ at 6 months without additional glaucoma procedures. Complications and need for additional procedures were rare. Phaco-KDB may be an effective and safe alternative to more invasive filtering surgery in many patients with severe glaucoma.

Keywords: glaucoma, goniotomy, IOP

\section{Introduction}

Glaucoma represents a leading cause of blindness, and projections estimate that its incidence will continue to increase. ${ }^{1}$ With increasing incidence comes increasing importance of effective and safe IOP-lowering therapies suitable for patients with all types and severities of disease, which would prevent progression of optic nerve damage and subsequent decline in quality of life. ${ }^{2}$ Traditionally, surgical procedures for the treatment of glaucoma have provided the greatest IOP reduction when compared to medications or laser treatment. ${ }^{3}$ Although effective, trabeculectomy and tube shunts have a higher risk of vision-threatening complications, including early hypotony and life-long risk of bleb or device-related complications. ${ }^{4}$ Lengthy visual recovery times, activity restriction, and need for frequent follow-up and repeat procedures in the early postoperative period also compromise patients' quality of life as well as health care 
efficacy and cost. Microinvasive glaucoma surgery (MIGS) refers to a group of ab interno procedures with a significantly lower risk of complications compared to traditional glaucoma surgery. These procedures serve as safe and effective alternatives that can be implemented earlier in the course of disease and may prevent or delay the need for more invasive and risky filtering or shunt surgeries.,

The Kahook Dual Blade (KDB; New World Medical, Rancho Cucamonga, CA, USA) is an ophthalmic surgical knife that can be used to perform ab interno trabeculectomy (commonly referred to as goniotomy). Since introduction of the KDB in 2015, a growing number of studies have demonstrated its efficacy and safety in reducing IOP and medication burden. ${ }^{7,8}$ Unlike traditional goniotomy, which is often performed in congenital glaucoma, the KDB's unique design allows a complete resection of diseased trabecular meshwork (TM) on the inner wall of Schlemm's canal (SC), allowing continuous flow of aqueous humor from the anterior chamber to the collector channel and distal pathway outflow system. ${ }^{9}$ The KDB has also been equipped with an excellent safety profile. The most common complications are IOP spikes secondary to steroid response and intraoperative or early postoperative blood reflux that is to be expected with unroofing of several collector channels and is generally transient. ${ }^{10}$

Indications for MIGS procedures, particularly SC- and suprachoroidal stents, have generally been restricted to mildto-moderate glaucoma, due to moderate efficacy compared to subconjunctival filtering procedures and perhaps also influenced by restrictive insurance coverage criteria. As a result, most studies on MIGS devices have been limited to these populations. However, a previous study on stand-alone KDB goniotomy has shown promising efficacy and safety in severe glaucoma patients. ${ }^{11}$ Another study demonstrated the efficacy of KDB combined with phacoemulsification (phaco-KDB) in glaucoma patients with multiple severities of which 22 had severe glaucoma. ${ }^{8}$ However, we are lacking peer-reviewed literature on the effects of phaco-KDB in patients specifically with severe glaucoma. To our knowledge, there is no study that has specifically examined the efficacy of KDB goniotomy combined with cataract surgery in patients with severe and refractory glaucoma at 6 months. In this study, we aimed to describe 6-month outcomes of goniotomy using the phaco-KDB in patients with severestage glaucoma and cataracts.

\section{Methods}

A retrospective review was performed of 42 eyes in 36 patients who received phaco-KDB at the Mason Eye
Institute at the University of Missouri between February 21, 2017, and January 23, 2018, after obtaining institutional review board (IRB) approval from the University of Missouri. All patients had severe-stage glaucoma, defined by ICD-10 as optic nerve abnormalities consistent with glaucoma, and glaucomatous visual field abnormalities in both hemifields and/or loss within $5^{\circ}$ of fixation in at least one hemifield. ${ }^{11,12}$ Included patients also had a minimum of 6 months of follow-up.

Exclusion criteria were as follows: any other procedure performed during the phaco-KDB and less than 6 months of follow-up. A de-identified data set was analyzed. Due to the retrospective nature of this study, the University of Missouri IRB granted a waiver of informed consent. All procedures performed in studies involving human participants were in accordance with the ethical standards of the institutional and/or national research committee and with the 1964 Declaration of Helsinki and its later amendments or comparable ethical standards.

\section{Surgical procedure}

All surgeries were performed by one surgeon at our outpatient surgery center. Topical anesthetic was instilled, and the eye was prepared and draped in a sterile manner. A Lieberman lid speculum was used to hold the eyelids open. A paracentesis incision was made, and the anterior chamber was filled with cohesive viscoelastic. A temporal near clear $2.4 \mathrm{~mm}$ incision was made into the anterior chamber. The patients' head and microscope were turned to allow proper visualization of the nasal angle with a Swan-Jacob gonio prism. The KDB was inserted into the anterior chamber, the tip of the instrument was used to make an initial incision into the TM, and then a strip of TM was excised circumferentially, spanning four clock hours. A strip of TM was removed with micro forceps or Utrata forceps. This was followed by standard phacoemulsification with IOL implantation. At the conclusion of the procedure, the eye was filled with balanced salt solution and intracameral Moxifloxacin to achieve an IOP in the mid-20s, in order to minimize early blood reflux. No viscoelastic was left in the anterior chamber.

\section{Postoperative care and follow-up}

Following surgery, topical broad-spectrum antibiotics were given for 1 week; prednisolone $1 \%$ was prescribed four times daily and then tapered over the next month. Patients continued their preoperative glaucoma medications postoperatively, and medications were reduced one class at a time only after the patient reached target IOP or lower. Patients 
were instructed to keep their head elevated, especially during sleep, and avoid straining, bending-over, and eye rubbing for the first 2 weeks following the surgery. No anti-coagulations were taken before or after surgery. All patients were seen day $1 \pm 1$ day, week $1 \pm 1$ week, month 1 , month 3 , and month 6. At each visit, data were collected on visual acuity, IOP, number of medications, gonioscopy findings, and complications related to surgery.

\section{Outcome measures}

The primary outcome was proportion of patients achieving IOP of $\leq 15 \mathrm{mmHg}$ at the 6-month postoperative follow-up without additional IOP-lowering procedures, including any laser. Secondary outcomes included IOP and medication reduction, and reduction in any complications or additional IOP-lowering procedures needed, including anterior chamber paracentesis. We recorded demographic data, baseline and postoperative IOP, number of glaucoma medications, postoperative additional IOP-lowering procedures, and any adverse events or interventions required. IOP spike was defined as elevation $>30 \mathrm{mmHg}$. Steroid response was defined as an IOP increase of $>25 \%$ from baseline while on topical prednisolone with minimum IOP of $28 \mathrm{mmHg}$ and subsequent reduction $>25 \%$ after discontinuing the topical prednisolone. ${ }^{13}$

\section{Statistical method}

Data were collected on the preoperative visit, postoperative day 1 , week 1 , month 1 , month 3 , month 6 , and month 12 . Due to the small sample size at 12 months, this time point was not considered for analysis. The data were analyzed using paired sample two-tailed $t$-tests to compare mean baseline and 6-month IOP, and the Wilcoxon signed-rank test to compare baseline and 6-month medications after checking assumptions. Tests were two-tailed with $\alpha=0.05$. We report all values as mean \pm SD for the data with normal distribution, median, and IQR for non-normally distributed values, and percentages (n) for categorical variables unless otherwise specified. We used SPSS version 24.0 (IBM Corporation, Armonk, NY, USA) for statistical analysis.

\section{Results}

The average age of the patients was $69.7 \pm 8.2$ years (range, 53-85). A total of 52.4\% (22/42) of evaluated eyes belonged to female patients and $47.6 \%(20 / 42)$ to male patients. The majority of patients in the study were Caucasian $(66.7 \%$, $28 / 42)$. In the sample, $64.3 \%(27 / 42)$ of eyes had primary open-angle glaucoma, $14.3 \%(6 / 42)$ had combined mechanism (phacomorphic angle closure with documented history of primary open-angle glaucoma), 14.3\% (6/42) had pseudoexfoliation, 4.8\% (2/42) had traumatic glaucoma, and 2.4\% (1/42) had normal-tension glaucoma. The baseline mean Humphrey visual field SITA-Standard 24-2 was $-13.1 \pm 8.5 \mathrm{~dB}$. A small number $(4.8 \% ; 2 / 42)$ of patients had undergone previous incisional surgeries (one patient had canaloplasty and cyclophotocoagulation and one had trabeculectomy and a tube).

The mean preoperative IOP was $17.1 \pm 4.8 \mathrm{mmHg}$ (range, 10-30 $\mathrm{mmHg}$ ) and mean (median [IQR]) baseline medication number was $2.4 \pm 1.3$ (2 [1.5]) (range, 0-5). Preoperative demographic and glaucoma information is reported in Table 1.

\section{Target IOP at 6 months}

At 6 months, $64.3 \%(27 / 42)$ of the eyes had achieved IOP $\leq 15 \mathrm{mmHg}$, and $45.2 \%$ (19/42) reached this target while also reducing medications (Table 2). A total of $31.0 \%(13 / 42)$ of eyes were able to achieve this target while also discontinuing all preoperative medications (mean of $2.2 \pm 1.2$ [2 (1)]).

Three patients in our study took no glaucoma medication preoperatively and all of them maintained IOP $\leq 18 \mathrm{mmHg}$ without medications postoperatively.

Table I Baseline demographic and glaucoma status data

\begin{tabular}{|c|c|}
\hline & KDB $(n=42)$ \\
\hline Age (years), mean $\pm S D$ & $69.7 \pm 8.2$ \\
\hline \multicolumn{2}{|l|}{ Gender, n (\%) } \\
\hline Female & $22(52.4)$ \\
\hline Male & $20(47.6)$ \\
\hline \multicolumn{2}{|l|}{ Ethnicity, n (\%) } \\
\hline Caucasian & $28(66.7)$ \\
\hline Black & $12(28.6)$ \\
\hline Asian & $\mathrm{I}(2.4)$ \\
\hline Hispanic & $\mathrm{I}(2.4)$ \\
\hline \multicolumn{2}{|l|}{ Glaucoma type, n (\%) } \\
\hline Primary open-angle & $27(64.3)$ \\
\hline Combined mechanism & $6(14.3)$ \\
\hline Pseudoexfoliative & $6(14.3)$ \\
\hline Traumatic & $2(4.8)$ \\
\hline Normal tension & $\mathrm{I}(2.4)$ \\
\hline Baseline visual field index (\%), mean \pm SD & $62.3 \pm 27.6$ \\
\hline Baseline mean deviation $(\mathrm{dB})$, mean $\pm \mathrm{SD}$ & $-13.1 \pm 8.5$ \\
\hline Baseline pattern $S D(d B)$, mean $\pm S D$ & $8.6 \pm 3.7$ \\
\hline Baseline IOP $(\mathrm{mmHg})$, mean \pm SD & $17.1 \pm 4.8$ \\
\hline Baseline medications, mean \pm SD & $2.4 \pm 1.3$ \\
\hline Previous incisional surgery, n (\%) & $2(4.8)$ \\
\hline
\end{tabular}

Abbreviation: KDB, Kahook Dual Blade. 
Table 2 Eyes with severe glaucoma achieving 6-month goals after phaco-KDB $(n=42)$

\begin{tabular}{|l|l|}
\hline IOP goal & $\%(\mathbf{n})$ \\
\hline$\leq 15 \mathrm{mmHg}$ & $64.3(27)$ \\
\hline$\leq 15 \mathrm{mmHg}+$ medication reduction & $45.2(19)$ \\
\hline$\leq 15 \mathrm{mmHg}+$ complete medication discontinuation & $31.0(13)$ \\
\hline
\end{tabular}

Abbreviation: phaco-KDB, Kahook Dual Blade combined with phacoemulsification.

\section{IOP reduction}

Mean IOP reduction was $2.1 \pm 4.7 \mathrm{mmHg}$, a significant $(P=0.022)$ reduction from mean baseline IOP of $17.1 \pm 4.8 \mathrm{mmHg}$ to $15.0 \pm 3.00 \mathrm{mmHg}$ at 6 months. Figure 1 shows the preoperative and 6-month IOP measurements for all eyes. IOP remained relatively stable throughout the postoperative period, with a small peak to near baseline at week 1 (Figure 2). Two eyes had a postoperative IOP $\leq 6 \mathrm{mmHg}$ on day 1 , which persisted in one eye for 1 month off all medications, without hypotony-related complications or evidence of cyclodialysis. After 3 months, no eyes had IOP $\leq 6 \mathrm{mmHg}$ or clinically significant hypotony.

\section{Medication reduction}

Mean medication reduction from baseline was $1.2 \pm 1.4$ (1 [2]), significantly reducing $(P<0.001)$ mean (median [IQR]) medication number from $2.4 \pm 1.3(2[1.5])$ at baseline to $1.2 \pm 1.4(1[2])$ at 6 months. From baseline, mean medication number trended down with a peak at 1 month postoperatively, reflecting responses to the change in IOP (Figure 3).

\section{Complications and additional procedures}

We recorded information on any adverse events at day 1 , week 1 , month 1 , month 3 , and month 6 postoperatively (Table 3). The majority of eyes (32/42) experienced microhyphema within the first week after surgery; no eyes experienced layered hyphema or hyphema persisting more than 2 weeks. No eyes with microhyphema required any specific treatment. During the 6-month timeframe, 7.1\% (3/42) of eyes had visually significant complications, defined as two or more lines of reduction in best corrected visual acuity compared to baseline. There were two instances of cystoid macular edema (CME) (4.8\%) and one case of epiretinal membrane (ERM) (2.4\%). One of two cases of CME resolved, and one case persisted through 6 months, along with the development of ERM. However, the patient remained at +0.1 logmar (20/25 Snellen) vision and did not require any additional surgery. At no time point did any eye have an IOP of $>30 \mathrm{mmHg}$.

A small number $(7.1 \% ; 3 / 42)$ of eyes required a further glaucoma procedure before 6 months (Table 4 ) due to insufficient IOP lowering or evidence of further glaucoma progression. These procedures consisted of one case of MicroPulse laser trabeculoplasty (MLT), one case of Xen ${ }^{\circledR}$ gel stent, and one case of Ahmed valve implantation. Only one of the eyes that required additional procedure experienced complications; the patient who received the Xen gel stent had an unusual history of persistent CME, which required multiple sub-Tenon's steroid injection followed by dexamethasone intravitreal implant.

\section{Discussion}

Excisional goniotomy performed using the KDB along with cataract surgery significantly reduced both mean IOP and number of medications compared to baseline in patients with severe-stage open-angle glaucoma. A substantial number (64.3\%) of eyes achieved a 6-month IOP lower than $15 \mathrm{mmHg}$, considered a reasonable target for most patients with severe-stage glaucoma.

While the mean observed IOP reduction of $2.1 \pm 4.7 \mathrm{mmHg}$ is less than that reported by Salinas et $\mathrm{al}^{10}$ in severe/refractory

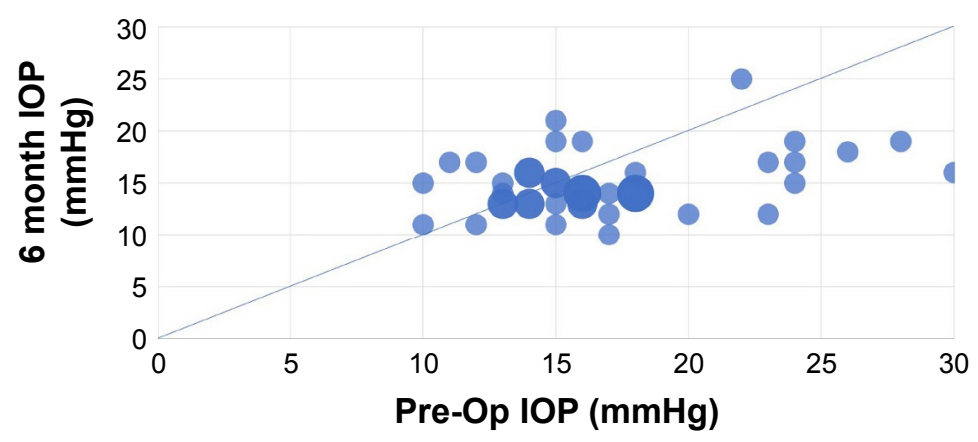

Figure I Scatter plot of preoperative and 6-month IOPs among patients $(n=42)$ who underwent phaco-KDB. Note: Bubble size is weighted by data point frequency.

Abbreviations: phaco-KDB, Kahook Dual Blade combined with phacoemulsification; Pre-Op, Preoperative. 


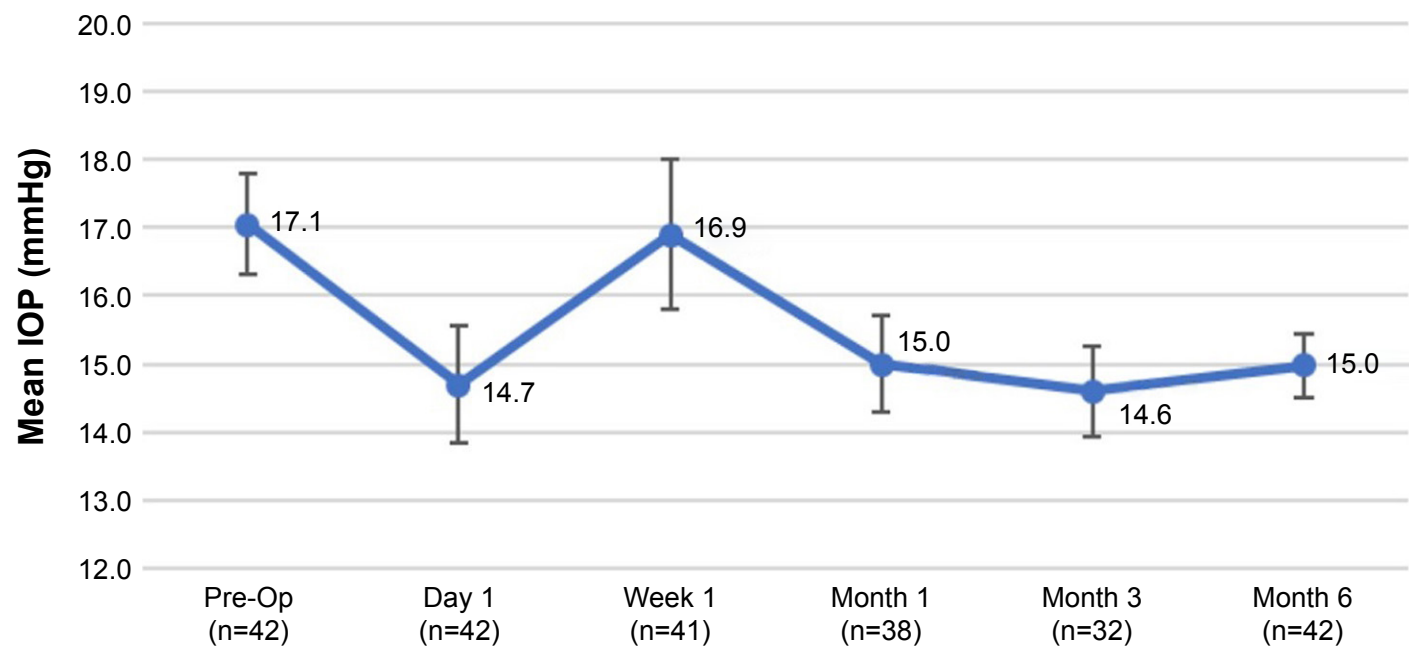

Figure 2 Mean $( \pm$ SEM) IOP reduction for phaco-KDB in severe glaucoma patients over the 6-month postoperative period. Abbreviations: phaco-KDB, Kahook Dual Blade combined with phacoemulsification; Pre-Op, Preoperative; SEM, standard error of the mean.

patients treated with stand-alone KDB goniotomy, the proportion of eyes achieving IOP $\leq 15 \mathrm{mmHg}$ in our study (64.3\%) was comparable to that reported by the same group (in which $63.5 \%$ of patients achieved IOP $\leq 14 \mathrm{mmHg}$ at 6 months). Similarly, Berdahl et al ${ }^{14}$ reported outcomes for stand-alone KDB goniotomy in primarily mild-to-moderate glaucoma patients (17\% had severe glaucoma), in which mean baseline IOP was significantly higher $(23.5 \mathrm{mmHg})$ than in our study $(17.1 \mathrm{mmHg})$, allowing more dynamic range for pressure reduction. However, at 6 months, the Berdahl et al's study population showed a mean IOP of $15.0 \mathrm{mmHg}$, comparable to our 6-month result of mean 15.0 $\pm 3.0 \mathrm{mmHg} .{ }^{14}$ It has been our experience that regardless of the level of baseline IOP, KDB goniotomy and other Schlemm's canal procedures achieve final IOP in the midteen range. Therefore, reporting of success defined by mean
IOP or percentage drop rather than final IOP achieved can lead to conflicting results.

A study by Ahmed et al ${ }^{15}$ using the Trabectome demonstrated a 6 -month IOP of $15.7 \pm 4.0 \mathrm{mmHg}$ and mean medications of $2.1 \pm 1.4$, versus our observation of $15.0 \pm 3.0 \mathrm{mmHg}$ and $1.2 \pm 1.4$ medications.

Hyphema after KDB goniotomy is an expected observation indicating patent outflow channels, representing reflux hemorrhage from the SC. Most eyes $(76.2 \%)$ in this study experienced microhyphema within the week following surgery, but none of our patients experienced layered hyphema at any time point or microhyphema lasting over 2 weeks. The authors believe that achieving an IOP in the mid-20s at the conclusion of the surgery and keeping the head elevated for the first 2 weeks following the surgery prevent significant hyphema events and speed up resolution. We do not hold

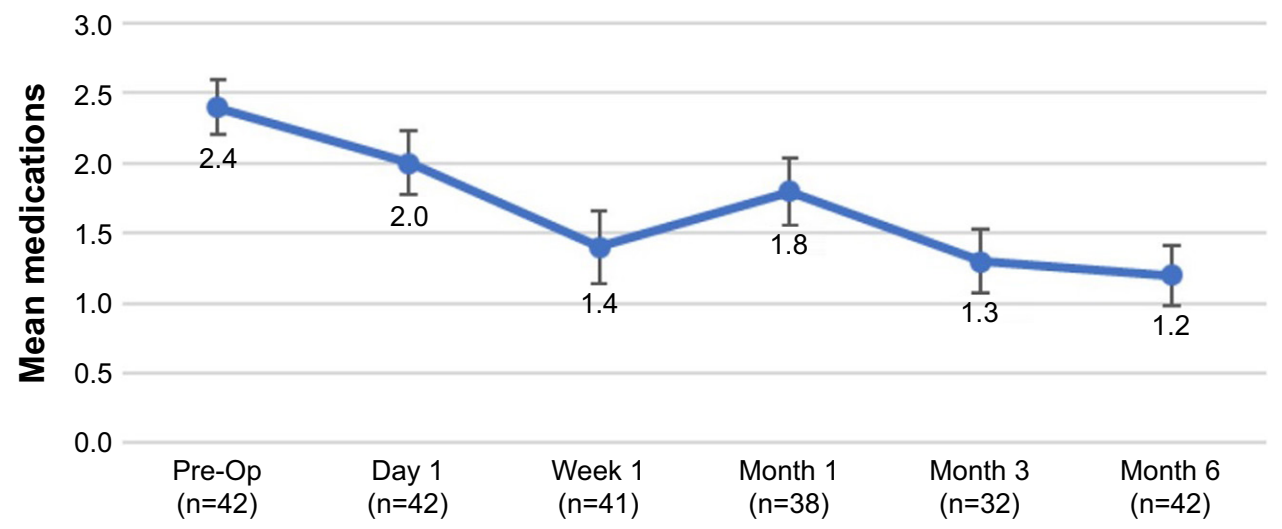

Figure 3 Mean $( \pm$ SEM) medication reduction for phaco-KDB in severe glaucoma patients during the 6-month postoperative period. Abbreviations: phaco-KDB, Kahook Dual Blade combined with phacoemulsification; Pre-Op, Preoperative; SEM, standard error of the mean. 
Table 3 Incidence of all adverse events for phaco-KDB patients with severe glaucoma $(n=42)$

\begin{tabular}{|l|l|l|l|l|l|}
\hline Adverse event & I day & I week & I month & 3 months & 6 months \\
\hline Microhyphema $^{\mathrm{a}}$ & $30(7 \mathrm{I} .4 \%)$ & $2(4.9 \%)$ & & & \\
\hline Hyphema $^{\mathrm{b}}$ & & & & & \\
\hline ERM & & I $(2.4 \%)$ & & & \\
\hline CME & & & $2(5.3 \%)$ & & \\
\hline
\end{tabular}

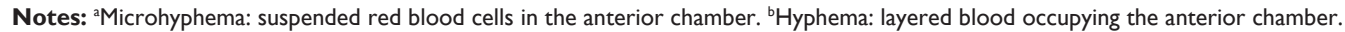

Abbreviations: CME, cystoid macular edema; ERM, epiretinal membrane; phaco-KDB, Kahook Dual Blade combined with phacoemulsification.

any anti-coagulants in patients undergoing phaco-KDB, or any other ab-interno Schlemm's canal procedure prior to or after surgery.

Visually significant adverse events such as CME and ERM were rare and not any more frequent than seen for phacoemulsification alone. ${ }^{16}$ None of our patients had IOP spikes, defined as IOP $>30 \mathrm{mmHg}$ following the procedure, at any time during the 6 month follow-up period. Considering the severity of their glaucoma, all patients kept the same number of medications until their IOP met their target, and then discontinued one class at a time with a shorter follow-up.

With this protocol, patients in this study were able to achieve an upward trend of success, allowing $69.0 \%$ of the cases to achieve target IOP of $\leq 15 \mathrm{mmHg}$ without requiring any additional procedure at 6 months. Less than $10 \%$ of the eyes experienced visually significant complications or required additional glaucoma procedures. Of the two patients who had prior glaucoma surgeries, one with a history of trabeculectomy and tube implant had a successful outcome at 6 months, whereas the patient with the canaloplasty and cyclophotocoagulation required a Xen ${ }^{\circledR}$ gel stent at 6 months.

Although these results reinforce the efficacy and safety of goniotomy using the KDB in patients with severe glaucoma, the small sample size and follow-up period are limitations of the study, along with its retrospective nature potentially associated with selection bias. Six patients contributed both eyes to the cohort, reducing variable independence. The types of glaucoma assessed were heavily biased toward primary open-angle glaucoma, with a few cases of other types. Therefore, we cannot confidently conclude that the
KDB procedure works equally well across all types of glaucoma. Since the KDB was only introduced at our center at the start of the study period, there may have been some learning effect, although the learning curve for this procedure was found to be relatively short and straightforward. We did not have a phacoemulsification-only control group that could demonstrate the additional IOP-lowering effect of KDB surgery beyond the known IOP-lowering effect of cataract surgery alone, as it is rare for patients in our practice with severe glaucoma to undergo stand-alone phacoemulsification. In the 2016 COMPASS trial looking at patients with mildto-moderate glaucoma, only $44 \%$ of the phaco-alone patients reached pressures of $18 \mathrm{mmHg}$ or less at either 12 months or 24 months. ${ }^{16}$ Moreover, in patients with any open-angle component and severe disease, the level of IOP reduction offered by phaco-alone would likely be insufficient.

Further studies with more robust sample sizes, longer follow-up period, a broader patient population, and prospective randomized controlled study design will help strengthen evidence for the appropriate use of phaco-KDB in patients with severe glaucoma. Here, we have demonstrated that excisional goniotomy with the KDB may serve as a safe and viable IOP-lowering alternative to more invasive filtering surgeries in patients with severe-stage glaucoma undergoing cataract surgery.

\section{Précis: achieved target IOP $\leq 15$ at 6 months}

Severe glaucoma patients achieved significant IOP and medication reductions and achieved target IOP at 6 months

Table 4 Incidence of all additional IOP-lowering procedures following phaco-KDB in patients with severe glaucoma ( $\mathrm{n}=42$ )

\begin{tabular}{|l|l|l|l|l|l|}
\hline Additional procedure & Day I & Week I & Month I & Month 3 & Month 6 \\
\hline Paracentesis & $2(4.8 \%)$ & $2(4.8 \%)$ & I (2.4\%) & & \\
\hline Ahmed valve & & & & I (2.4\%) & \\
\hline Xen $^{\circledR}$ gel stent & & & & & I (2.4\%) \\
\hline MLT & & & & & I (2.4\%) \\
\hline
\end{tabular}

Abbreviations: MLT, MicroPulse Laser Trabeculoplasty; phaco-KDB, Kahook Dual Blade combined with phacoemulsification. 
following phaco-KDB. Rates of complications or need for further glaucoma procedures were low.

\section{Acknowledgments}

The authors acknowledge Amy Folkerts and Karen Langenberg for their professional editing and manuscript submission support. New World Medical provided the editing support and processing fee for this study. The abstract was presented at World Glaucoma Congress, Melbourne, Australia, March 27, 2019.

\section{Disclosure}

Dr An has served as a consultant for New World Medical. No personal fees or research support had been received during the conduct of the study. The authors report no other conflicts of interest in this work.

\section{References}

1. Tham Y, Li X, Wong T, Quigley H, Aung T, Cheng C. Global prevalence of glaucoma and projections of glaucoma burden through 2040: a systematic review and meta-analysis. Ophthalmology. 2014;121(11): 2081-2090. doi:10.1016/j.ophtha.2014.05.013

2. Quaranta L, Riva I, Gerardi C, Oddone F, Floriano I, Konstas A. Quality of life in glaucoma: a review of the literature. Adv Ther. 2016;33: 959-981. doi:10.1007/s12325-016-0333-6

3. Lichter P, Musch D, Gillespie B, et al. Interim clinical outcomes in the collaborative initial glaucoma treatment study comparing initial treatment randomized to medications or surgery. Ophthalmology. 2001;108(11): 1943-1953.

4. DeBry P, Perkins T, Heatley G, Kaufman P, Brumback L. Incidence of late-onset bleb-related complications following trabeculectomy with mitomycin. Arch Ophtholmol. 2002;120(3):297-300. doi:10.1001 archopht.120.3.297
5. Pillunat L, Erb C, Jünemann A, Kimmich F. Micro-invasive glaucoma surgery (MIGS): a review of surgical procedures using stents. Clin Ophthalmol. 2017;11:1583-1600. doi:10.2147/OPTH.S135316

6. Chen DZ, Sng CA. Safety and efficacy of microinvasive glaucoma surgery. J Ophthalmol. 2017;Article ID 3182935, 13 pages. doi: $10.1155 / 2017 / 3182935$

7. Dorairaj S, Seibold L, Radcliffe N, et al. 12-month outcomes of goniotomy performed using the kahook dual blade combined with cataract surgery in eyes with medically treated glaucoma. Adv Ther. 2018;35(9):1460-1469. doi:10.1007/s12325-018-0755-4

8. Greenwood M, Seibold L, Radcliffe N, et al. Goniotomy with a singleuse dual blade: short-term results. J Cataract Refract Surg. 2017;43(9): 1197-1201. doi:10.1016/j.jcrs.2017.06.046

9. Seibold L, Soohoo JR, Ammar DA, Kahook MY. Preclinical investigation of ab interno trabeculectomy using a novel dual-blade device. Am J Ophthalmol. 2013;155(3):524-529. doi:10.1016/j.ajo.2012. 09.023

10. Salina L, Chaudhary A, Berdahl J, et al. Goniotomy using the Kahook Dual Blade in severe and refractory glaucoma: six month outcomes. J Glaucoma. 2018;27(10):849-855.

11. Mattox C. Glaucoma stage definitions. 2014. Available from: https:// www.aao.org/assets/68cdbc7d-35ba-47ce-b95e-726eb503fe3e/ 635577092075270000/how-to-code-for-different-stages-of-glaucomausing-icd-10-codes-pdf?inline=1. Accessed January 15, 2019.

12. Mills R, Budenz D, Lee $P$, et al. Categorizing the stage of glaucoma from pre-diagnosis to end-stage disease. Am J Ophthalmol.2006;141(1):24-30. doi:10.1016/j.ajo.2005.07.044

13. Chang D, Tan J, Tripodis Y. Risk factors for steroid response among cataract patients. J Cataract Refract Surg. 2011;37(4):675-681. doi:10. 1016/j.jcrs.2010.10.051

14. Berdahl J, Gallardo M, ElMallah M, et al. Six-month outcomes of goniotomy performed with the Kahook Dual Blade as a stand-alone glaucoma procedure. Adv Ther. 2018;35(11):2093-2102. doi:10.1007/ s12325-018-0803-0

15. Ahmed S, Bhatt A, Schmultz M, Mosaed S. Trabectome outcomes across the spectrum of glaucoma disease severity. Graefes Arch Clin Exp Ophthalmol.2018;256(9):1703-1710. doi:10.1007/s00417-018-4023-8

16. Vold S, Ahmed I, Craen ER, et al. Two-year COMPASS trial results: supraciliary microstenting with phacoemulsification in patients with open-angle glaucoma and cataracts. Ophthalmology. 2016;123(10): 2103-2112. doi:10.1016/j.ophtha.2016.06.032
Clinical Ophthalmology

\section{Publish your work in this journal}

Clinical Ophthalmology is an international, peer-reviewed journal covering all subspecialties within ophthalmology. Key topics include: Optometry; Visual science; Pharmacology and drug therapy in eye diseases; Basic Sciences; Primary and Secondary eye care; Patien Safety and Quality of Care Improvements. This journal is indexed on

\section{Dovepress}

PubMed Central and CAS, and is the official journal of The Society of Clinical Ophthalmology (SCO). The manuscript management system is completely online and includes a very quick and fair peer-review system, which is all easy to use. Visit http://www.dovepress.com/ testimonials.php to read real quotes from published authors. 